\title{
Obstacles Affect Perceptions of Egocentric Distances in Virtual Environments
}

\author{
Daisuke Mine ${ }^{1 *}$, Sakurako Kimoto ${ }^{2}$ and Kazuhiko Yokosawa ${ }^{2}$ \\ ${ }^{1}$ Graduate School of Interdisciplinary Information Studies, The University of Tokyo, Tokyo, Japan, ${ }^{2}$ Department of Psychology, \\ The University of Tokyo, Tokyo, Japan
}

Distance perception in humans can be affected by oculomotor and optical cues and a person's action capability in a given environment, known as action-specific effects. For example, a previous study has demonstrated that egocentric distance estimation to a target is affected by the width of a transparent barrier placed in the intermediate space between a participant and a target. However, the characteristics of a barrier's width that affect distance perception remain unknown. Therefore, we investigated whether visual and tactile inputs and actions related to a barrier affect distance estimation to a target behind the barrier. The results confirmed previous studies by demonstrating that visual and tactile

OPEN ACCESS

Edited by:

Richard Skarbez,

La Trobe University, Australia

Reviewed by:

Gurjot Singh,

Fairleigh Dickinson University,

United States

Jerald Thomas,

University of Minnesota Twin Cities,

United States

*Correspondence:

Daisuke Mine

mine@cyber.t.u-tokyo.ac.jp

Specialty section:

This article was submitted to Virtual Reality and Human Behaviour,

a section of the journal

Frontiers in Virtual Reality

Received: 16 June 2021

Accepted: 31 October 2021

Published: 16 November 2021

Citation:

Mine $D$, Kimoto $S$ and Yokosawa $K$ (2021) Obstacles Affect Perceptions of

Egocentric Distances in Virtual Environments.

Front. Virtual Real. 2:726114.

doi: 10.3389/frvir.2021.726114 presentations of the barrier's width affected distance estimation to the target. However, this effect of the barrier's width was not observed when the barrier was touchable but invisible nor when the barrier was visible but penetrable. These findings indicate the complexity of action-specific effects and the difficulty of identifying necessary information for inducing these effects.

Keywords: action-specific effects, distance perception, spatial perception, vision, touch

\section{INTRODUCTION}

We assume that our perception of the environment is precise and wholly dependent on visual inputs, including oculomotor and optical cues. However, several studies have demonstrated that a person's action capability influences spatial perception. Bhalla and Proffitt. (1999) demonstrated that a person's ability to ascend a hill determined by carrying a heavy backpack or being tired affects their estimate of the slope's angle. Proffitt et al. (2003) reported that people carrying a heavy backpack perceive objects as farther away than those without a heavy backpack. Witt et al. (2005) showed that participants estimated a target as being close when holding a hand-held tool than not holding one. These studies suggest that the perception of geometrical properties of space is determined not only by visual inputs but also by the energy costs of activities in that space. Other studies have shown that the size of a participant's body influences the egocentric distance estimation to an object and the size estimation of the object, which might be explained by different energy costs of intended actions depending on the body size (van der Hoort et al., 2011; Ogawa et al., 2017; Tosi et al., 2020; see also; Mine et al., 2020). Several other studies have provided evidence of similar action-specific effects on distance perception (see Creem-Regehr and Kunz, 2010; Philbeck and Witt, 2015, for a review). These studies have used a variety of experimental paradigms and manipulated constraints or extensions of intended actions, including the effort needed for action (Witt et al., 2011; Kirsch et al., 2012), weight on the body (Lessard et al., 2009), and tool-use 
(Witt and Proffitt, 2008; Witt, 2011; Costello et al., 2015). Furthermore, the condition of the perceiver and the condition of the space affects the perception of geometrical properties. Morgado et al. (2013) set a transparent barrier in the intermediate space between the participants and a target and asked the participants to estimate the egocentric distance to the target after imagining that they are reaching around the barrier to the target. The results indicated that participants showed a more extended distance estimation when the width of the transparent barrier was wide than when it was narrow. Therefore, varying the action cost of reaching around a barrier by manipulating its width affected the distance perception to the target placed behind the barrier.

Visual inputs play a crucial role in perceiving the space around a person. However, other sensory inputs also provide cues on a person's surroundings and possible actions in the given space. The studies of blind people (including late blind) and blindfolded sighted people have provided evidence that people can perceive their surroundings using sensory inputs other than vision (Lahav and Mioduser, 2008; Afonso et al., 2010). Especially, tactile inputs are direct cues of a person's action space. For example, if a person tries to extend an arm straight towards a target and touch something in the way, he/she will know that the arm cannot be extended any further in that direction, even if there is no visual obstacle. Moreover, if a visual obstacle is penetrable (there are no tactile sensations from the obstacle), the person could ignore the obstacle and extend her/his arm straight at a target even though the person can see an obstacle between him/her and the target. Therefore, tactile inputs are direct cues of the spatial limitation of a person's physical actions, although objects that are transparent but touchable or visible but penetrable are rare in the real world. Tactile information about an obstacle might distort the perception of egocentric distance to a target even when visual information was missing if distance perception were influenced by recognizing the presence of obstacles located in intermediate space between a target and a perceiver.

The present study was designed to investigate whether the egocentric distance is affected by tactile information about obstacles presented at the intermediate space between a perceiver and a target without any visual information about the obstacle. Most previous studies investigating action-specific effects on perception have focused on visual information from space. However, tactile inputs have a critical role in perceiving the surroundings, especially obstacles that physically limit the person's action possibilities. Therefore, tactile inputs of obstacles might affect the perception of egocentric distance. This study also investigated whether the findings of Morgado et al. (2013) could be replicated. Experimental findings on actionspecific effects have replicability problems. Several studies have failed to replicate action-specific effects (e.g., Hutchison and Loomis, 2006). Quarona et al. (2020) demonstrated that obstacles in front of a target delay shape judgments of the target object behind the barrier. Nevertheless, the effects of obstacles on distance perception have not been replicated. The results of Morgado et al. (2013) are surprising findings indicating that the surrounding environment's condition affects a person's perceptions, and we considered it essential to verify the robustness of this finding. Therefore, in Experiment 1, we developed a similar virtual environment to the study by Morgado et al. (2013).

\section{GENERAL METHODS}

\section{Participants}

We recruited healthy right-handed participants with normal or corrected-to-normal vision $(N=65)$. Of these, 36 (10 women; mean age, 21.8 years; age range, 19-26 years) participated in Experiment 1 as non-paid volunteers, and 29 (13 women; mean age, 22.7; age range, $19-45$ years) participated in Experiment 2 as paid volunteers. All the participants gave their written informed consent before participating in the experiments. The experiments and procedures described below were approved by the ethics committee of the University of Tokyo, Department of Psychology. All the experiments were conducted according to the principles and guidelines of the Declaration of Helsinki.

\section{Apparatus and Stimuli}

The participants saw a virtual environment through a headmounted display (HMD: Oculus Rift S, showing a stereoscopic image at a resolution of $2,560 \times 1,440$ ). The virtual environment was developed using Unity3 $\mathrm{D}$ and run on a Windows PC (ALIENWARE; Intel core $\mathrm{i} 7-8750 \mathrm{H}$ at $22 \mathrm{GHz}, 32 \mathrm{~GB}$ RAM, and NVIDIA GeForce RTX 2070). A pen-type device, 3D Systems Touch (3D Systems), consisting of a manipulator connected to an arm, was used to present tactile sensation (Figure 1A). The device generated tactile feedback about the surface of a virtual object when participants touched the virtual object with its pen tip. In addition, we used a Leap Motion Controller (Ultra leap, ltd.) as a sensor to track the participant's actual right-hand movements and synchronize these movements with those of a right-hand avatar displayed in the virtual environment.

The participants performed a haptic task and a distance estimation task in the experiments. In the haptic task, participants moved the pen-like haptic presentation device from side to side. In Experiment 1, the pen-like manipulator generated haptic feedback about the surface of the barrier when its tip encountered either a visible or an invisible barrier placed in front of the participants in the virtual environment (Figure 1). The barrier had a height of $24 \mathrm{~cm}$ and a width of either 10 or $30 \mathrm{~cm}$. In Experiment 2, a visible barrier was presented, with no haptic feedback generated when the manipulator encountered the barrier. The participants performed a distance estimation task after recognizing the barrier and its width using the manipulator. The participants adjusted a black circle by pressing a key with their left index and middle fingers until the distance between the black circle and a red reference point matched the distance between a target positioned behind the barrier and the reference point (Figure 2). In the distance estimation task, a right-hand avatar was presented in the virtual environment and moved synchronously with the participant's right-hand movement. The participants kept their right index finger on 

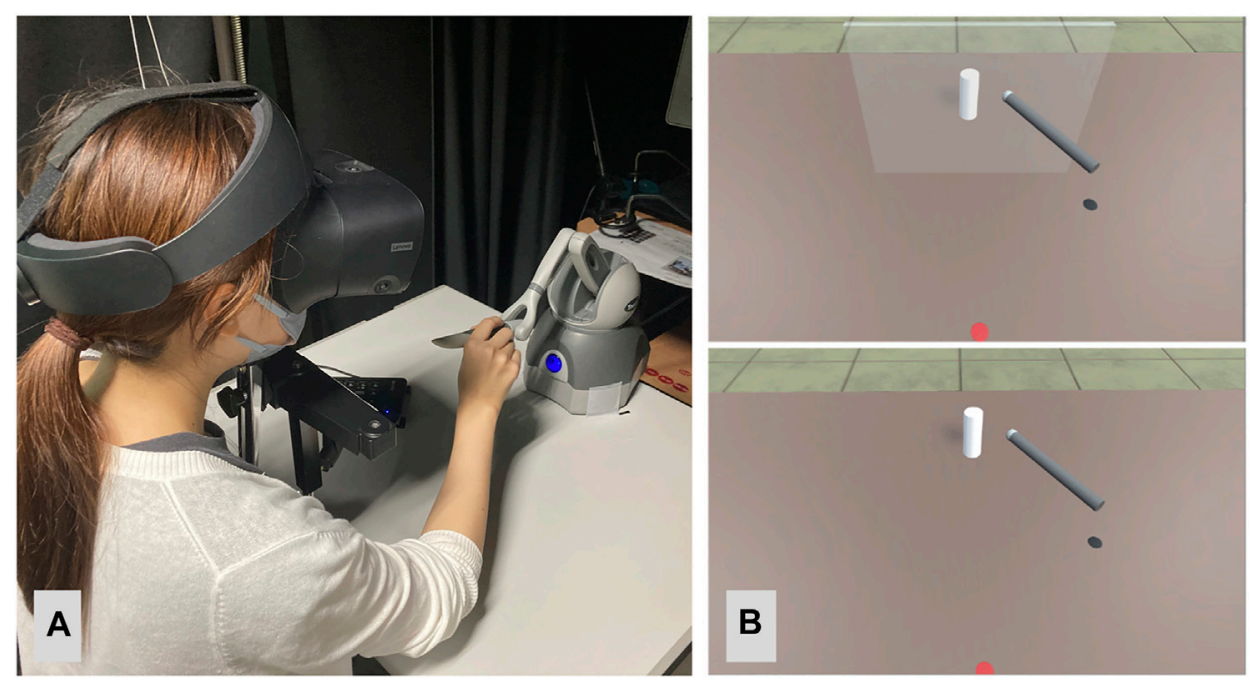

FIGURE 1 | The haptic task. The left figure shows the haptic task environment (A). Participants controlled a pen-like manipulator and tactilely recognized the presence and width of a barrier. The two figures on the right show the virtual environment that the participant saw through the HMD in the haptic task (B). The grey pen presented in the virtual environment moved synchronously with the manipulator. The upper figure shows the visible barrier condition, and the bottom figure shows the invisible barrier condition. Haptic feedback was generated when the grey pen encountered the visible or invisible barrier in Experiment 1 , and no haptic feedback was generated when the pen encountered the visible barrier in Experiment 2.
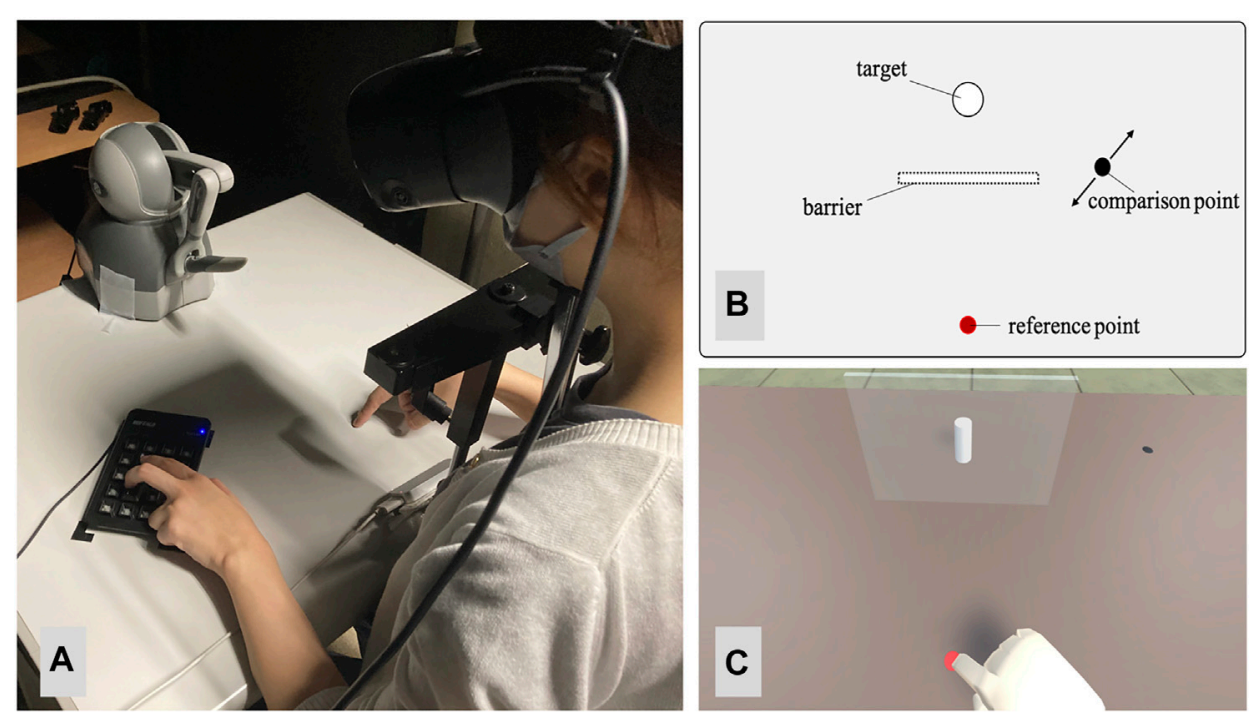

FIGURE 2 | The distance estimation task. In this task, a virtual right-hand avatar that moved synchronously with the participant's right hand was presented in the virtual environment. The participants put their right index finger on the red reference point and estimated the distance between the reference point and the target. Then, they adjusted the comparison point until the distance between the reference point and the comparison point matched the distance between the reference point and the target.

the red circle presented on their sagittal axis, $5 \mathrm{~cm}$ from the end of the table where they were seated. A white cylinder (height: $9 \mathrm{~cm}$, diameter: $3.2 \mathrm{~cm}$ ) was used as the target was positioned either $34 \mathrm{~cm}, 37 \mathrm{~cm}, 40 \mathrm{~cm}$, or $43 \mathrm{~cm}$ from the participant's right index finger, and the barrier was presented $24 \mathrm{~cm}$ from the participant's right index finger. The target cylinder and the barrier were aligned on the participant's sagittal axis. The black circle, which moved on a diagonal line of $45^{\circ}$ in the participant's right hemifield, was used as the comparison point. The initial position of the comparison point was either 24 or $53 \mathrm{~cm}$ from the participant's right index finger. These tasks are modified versions of those used in Morgado et al. (2013).

Before the experiment started, the participants watched a prerecorded video through their HMD showing a virtual hand 


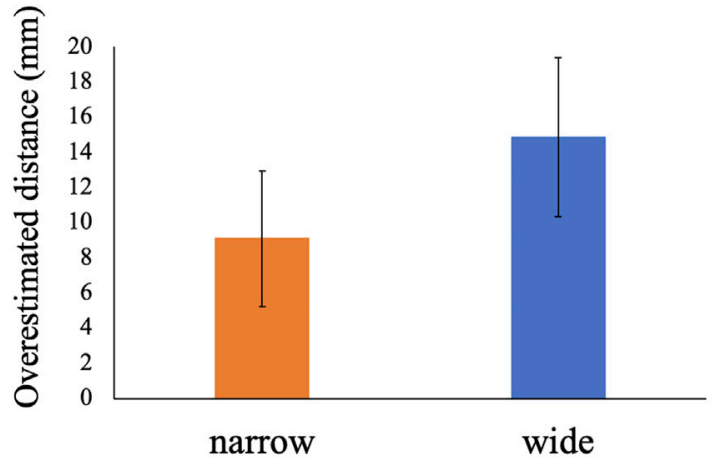

Visible condition

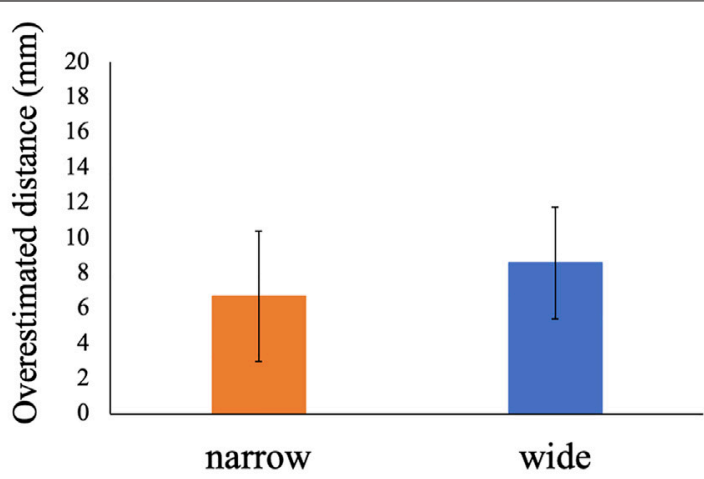

Invisible condition

FIGURE 3 | The mean of the participant's distance overestimation under each condition in Experiment 1. The left figure shows the visible condition results, and the right figure shows the invisible condition results. The overestimated distance was defined as the difference in the physical and the estimated distance between the reference point and the target. A positive value indicates distance overestimation. Error bars show the standard error.

reaching and grasping a target cylinder, which was shown to avoid discrepancies in imagined action between participants. The video showed a virtual hand reaching around the barrier in experiment 1 , while showed a virtual hand reaching straight to the target (i.e., penetrating the barrier) in Experiment 2.

\section{EXPERIMENT 1}

\section{Stimuli and Procedure}

The participants were seated at one end of a table placed in a laboratory. They were instructed to adjust the fit of their HMD and hold the manipulator of the pen-like haptic input device in their right hand. They also positioned their face on a chin rest attached to the table to prevent the head from moving during the experiment. At first, the participants watched a video through their HMD showing a virtual hand reaching around a $20 \mathrm{~cm}$ wide barrier and grasping a target cylinder. After watching the video, HMD showed the virtual world where the participants conducted tasks. A virtual table was placed in the virtual environment facing the participants, and a grey pen that was programmed to move synchronously with the pen-like manipulator, the target, and the barrier were presented on the virtual table (Figure 1B). The participants were asked to move the pen-like manipulator, tracing the barrier for several tens of seconds, to recognize the barrier's edges.

After the haptic task, the pen-like manipulator was removed. The virtual right-hand avatar appeared, and the participants were instructed to put their right index finger on the reference point in the virtual environment. Then, the distance estimation task started (Figure 2). The participants adjusted the black circle until the distance between the black circle and a red reference point matched the distance between a target positioned behind the barrier and the reference point. The participants were instructed to imagine the movement of reaching around the barrier at the target before estimating the distance to the target. When the participants finished adjustment, the experimenter recorded the distance. Then, the subsequent trial's haptic task started.

Each participant performed one practice block of eight trials followed by four experimental blocks, each of eight trials. A practice block was conducted with no barrier presented (i.e., no visual or tactical feedback) to enable participants to practice the visual matching task. In the visual matching task, they were instructed to adjust the comparison point until the distance between the comparison point and the reference point matched the distance between the target and the reference point. The experimental blocks consisted of the visible barrier (the visible condition) and the invisible barrier (the invisible condition) conditions. Each of these conditions consisted of a narrow barrier block and a wide barrier block. The order of the two conditions and the two blocks in each condition was pseudorandomized across participants. Tactile feedback was presented through the haptic presentation device in all the blocks.

\section{Results and Discussion}

The overestimated distance was defined as the difference between the estimated distance and the physical distance between the reference point and the target. Figure 3 indicates the results of Experiment 1. We compared the overestimated distance for narrow and wide barriers under visible and invisible barrier conditions. We checked normality of the data by KolmogorovSmirnov test, then conducted paired t-tests for the visible and invisible conditions respectively. A wide visible barrier resulted in participants estimating a longer distance to the target than a narrow visible barrier (narrow: mean $=9.09 \mathrm{~mm}, \mathrm{SD}=23.09 \mathrm{~mm}$; wide: mean $=14.86 \mathrm{~mm}, \mathrm{SD}=27.15 \mathrm{~mm})$, and a paired $t$-test indicated there is significant difference between them $(t(35)=$ 2.29, $p=0.028$, Hedge's $g=0.381$ ), which corroborated the previous findings by Morgado et al. (2013). On the other hand, there was no significant difference in the estimated distance between a narrow and a wide invisible barrier $(t(35)=0.70$, $p=0.489$, Hedge's $g=0.117$; narrow: mean $=6.69 \mathrm{~mm}, \mathrm{SD}=$ $22.18 \mathrm{~mm}$; wide: mean $=8.59 \mathrm{~mm}, \mathrm{SD}=18.96 \mathrm{~mm}$ ). The effect of 
tactile input about obstacles presented without any visual input on egocentric distance estimation to the target was not observed, suggesting that distance perception is affected by the width of a visible and touchable barrier but not by the width of an invisible but touchable barrier. Participants could realize the presence of the barrier even when it was invisible and understand that they cannot reach straight to the target because of the barrier. Moreover, they were instructed to imagine reaching around the barrier at the target before making distance estimations under both conditions. Nevertheless, recognizing the barrier's existence and its width merely through tactile inputs was insufficient to induce action-specific effects.

A possible explanation of this result is that action-specific effects on distance estimation rely entirely on the energy cost of action planning using visual information about a given space. Visually presented objects indicate particular action possibilities and the scale of the surroundings for conducting those actions (Proffitt and Linkenauger, 2013). Therefore, it is a possibility that visually presented barriers automatically, and perhaps unconsciously, induce action plans for reaching around the barrier. Specific studies have suggested that experimental processes of either motor experience or motor preparation are necessary to induce action-specific effects (e.g., Linkenauger et al., 2015). However, a Bayesian meta-analysis by Molto et al. (2020) indicated strong evidence of no difference in action-specific effects between conditions with and without motor experience or motor preparation. Therefore, there is a possibility that distance perception could be modulated only by visual feedback about barriers at the time of distance estimation, regardless of whether the barrier is touchable or not or the participant's actions before making estimations. To examine this possibility, we investigated the effect of a visible but untouchable barrier on distance perception in Experiment 2.

\section{EXPERIMENT 2}

Experiment 2 investigated the effect of visual information about an obstacle on making distance estimations. The experimental design was nearly identical to that of Experiment 1, except that the barrier in Experiment 2 was visible but untouchable. Participants were asked to imagine reaching the target by directly penetrating the barrier before making each distance estimation. If the distance estimation to the target was affected only by visual information about the barrier at the time of estimating, then neither the imagined action before estimating nor the ability to touch the barrier was expected to have a critical effect on the distance estimation.

\section{Stimuli and Procedure}

The stimuli and procedure of Experiment 2 were nearly identical to Experiment 1 except that the barrier was visible but untouchable (i.e., no tactile feedback was presented through the haptic presentation device) and the participants were instructed to imagine reaching straight at the target before estimating the distance. Before the experiment started, participants had watched a video through their HMD showing

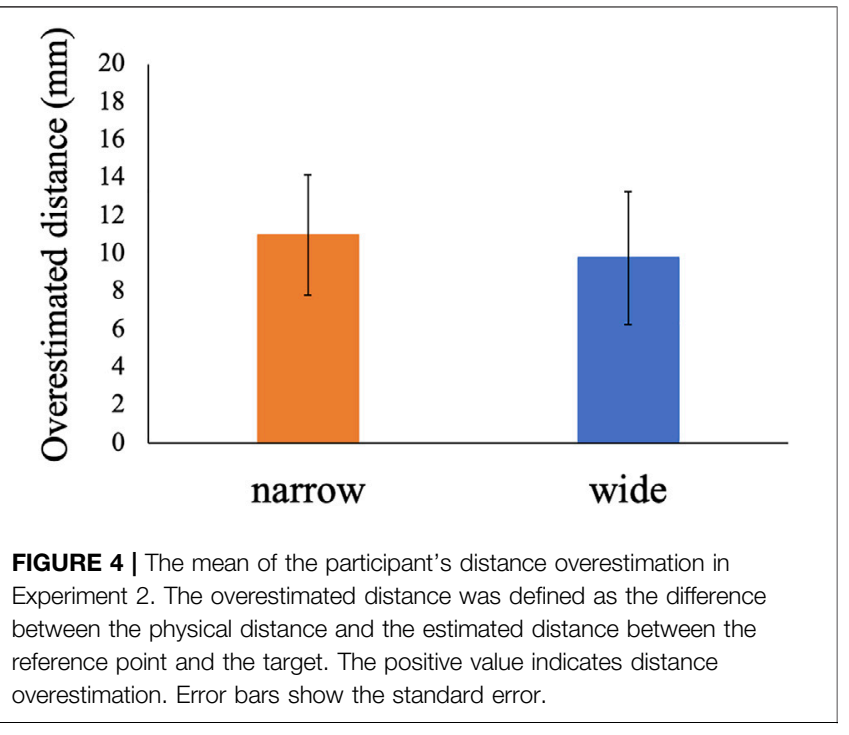

a virtual hand reaching a target directly by penetrating a barrier and grasping the target cylinder. Each participant performed one practice block of eight trials followed by two experimental blocks, each of eight trials. The barrier width was either narrow or wide in each experimental block. The order of the blocks was pseudorandomized across participants.

\section{Results and Discussion}

The overestimated distance was defined as the difference in the estimated distances and the physical distance between the reference point and the target. We compared the overestimated distance for the narrow and the wide barrier. We checked normality of the data by Kolmogorov-Smirnov test, then conducted a paired $t$-test. The results of Experiment 2 are shown in Figure 4.

A paired $t$-test indicated no significant differences in the estimated distance between a wide and a narrow barrier $(t$ $(28)=0.49, p=0.625$, Hedge's $g=0.092$; narrow: mean $=$ $10.99 \mathrm{~mm}, \mathrm{SD}=17.07 \mathrm{~mm}$; wide: mean $=9.79 \mathrm{~mm}, \mathrm{SD}=$ $18.80 \mathrm{~mm}$ ), suggesting that significant action-specific effects were not induced by the visually presented, untouchable barrier when the participants imagined the movement of directly reaching at the target before distance estimation. The results of Experiment 2 suggested that the penetrable barrier and imagining reaching straight at the target reduced the effect of the barrier width. Most previous studies investigating action-specific effects instructed participants to conduct or imagine a specific action while simultaneous visual information about the participant's surroundings automatically affords action possibilities. The action instructed by experimenters and the action afforded by visual stimuli were generally identical. For example, in Morgado et al., 2013, the participants were asked to imagine the action of reaching around a barrier in front of a target, while visual information of visible barrier also afforded the same action. If instructed and afforded actions are identical, it is impossible to distinguish which action affects the geometrical perception. However, the instructed and afforded actions are 
different in Experiment 2 of the present study. Visual stimuli of visible barrier afforded the movement of reaching around the barrier; on the other hand, the participants were instructed to imagine reaching straight toward the target by penetrating the barrier. The present results indicated that the instruction to imagine an action might overcome the limitations on action possibilities imposed by a visible barrier, and the effect of the barrier's width disappeared. Therefore, the action that participants imagined before estimating the distance was a critical factor in the action-specific effect.

\section{GENERAL DISCUSSION}

\section{Summary of the Results}

In Experiment 1, participants estimated the target as further away when a wide compared to a narrow barrier was visually and tactilely presented in the space between the participants and the target. In contrast, this effect was not observed for a tactilely but not visually presented barrier's width. Thus, only tactile information of the barrier width was insufficient to induce action-specific effects. One possible explanation of this result is that action-specific effects are related to energy costs of actions potentially determined through visual information about the space surrounding a person. However, in Experiment 2, the effect of a visually presented and penetrable barrier on distance estimation was not observed. Therefore, it seems that visual information of obstacles is insufficient to influence distance perception. These results suggest that visual inputs and action imagery of reaching for the barrier are necessary to induce actionspecific effects.

\section{Contributions of Vision, Touch, and Motor Intention}

The results of this study suggest that action-specific effects are complex and not amenable to simple explanations based on the effect of a single factor such as vision, touch, or motor intention. However, these results clarify the contributions of sensory inputs and motor intentions on distance estimation. Firstly, the results indicate that visual information is a crucial factor in action-specific effects. Experiment 1 showed that participants estimated a longer distance for a wide barrier that was visible and touchable, but not when the barrier was touchable but invisible. Visual information provides relatively accurate spatial information about our surroundings and supports movements requiring low energy (Cohen et al., 2010). In contrast, the results of Experiment 2 suggested that visual information by itself is insufficient to induce action-specific effects.

Combining the results of the two experiments of this study suggest that instructed motor imagination affects action-specific effects, at least when there is a conflict between instructed action and potential action afforded by visual stimuli. However, whether instructed action experiences (or imagination) affect actionspecific effects when the action instructed by the experimenter and the action afforded by visual information about the surroundings are identical remain to be identified (see Molto et al., 2020).

Tactile feedback about the barrier width did not induce significant action-specific effects in Experiment 1. Possibly, participants failed to recognize the difference in width between the narrow and wide barriers. As a result, the imagined motion trajectories might have been similar regardless of narrow or wide barriers. However, we believe this was not the case because the difference in the barrier's width was very large (10 vs $30 \mathrm{~cm}$ ). Moreover, Cohen et al. (2010) indicated that people take a more roundabout trajectory when reaching over obstacles to avoid a collision if the obstacle's height is uncertain. Suppose width perception through tactile inputs is uncertain in the invisible condition of Experiment 1 . In that case, the imagined action trajectory might become more roundabout in the invisible than in the visible condition, resulting in a higher overestimation of the target position in the invisible condition. However, the estimated distance was smaller in the invisible condition than in the visible condition (Figure 3), suggesting that width perception through tactile inputs might have been relatively accurate. If participants could recognize the barrier's width with accuracy just through tactile input, why the effect of the barrier's width on distance estimation was not observed in the invisible condition of Experiment 1? It is possible that motor planning relies more on visual than tactile inputs because visual inputs have a higher spatial resolution than tactile inputs. Previous studies have suggested that tactile inputs can indicate surrounding spatial information when participants are blind or blindfolded (see Morash et al., 2012, for review). However, in the invisible condition of Experiment 1, participants saw the visual stimuli displaying no barrier, and they were not blindfolded. Therefore, the planned action might be affected by visual information that there is no barrier in front of the target, resulting in less roundabout trajectory. Based on this interpretation, we can experimentally investigate the actual effects of spatial cognition through tactile inputs in an experiment; for example, blindfolded participants reach around a tactilely presented barrier to reach a target indicated by auditory stimuli.

\section{Replicability of Action-specific Effects}

As discussed in the introduction, experimental findings of action-specific effects have a replicability problem. Several studies have reported replication failures of action-specific effects. For example, Hutchison and Loomis. (2006) failed to replicate the findings of Proffitt et al. (2003), showing that people carrying a heavy backpack perceive a longer distance to a target than people not carrying a backpack (see also Proffitt et al., 2006, as Proffitt and other's response). Woods et al. (2009) also failed to replicate the findings of Proffitt et al. (2003) and Witt et al. (2004). However, it should be noted that there were specific methodological and theoretical differences between the original and replication studies. Molto et al. (2020) conducted a meta-analysis on action-specific effects and 
reported a small action-specific effect. However, if there might be a publication bias, it leads to overestimating the effect size of action on geometrical perception. Therefore, the effect of action and action planning on geometrical perception remains controversial. As a result, the critical method of validating actual action effects is reporting direct or conceptual replications, whether positive or negative. To the best of our knowledge, there are no replication studies on the effect of a barrier's width on egocentric distance perception. Experiment 1 successfully replicated the results by showing that the width of a visually and tactilely presented barrier affected the perception of egocentric distance. The effect size of the barrier's width in the visible condition of Experiment 1 was a very large effect according to the new effect size criteria specific to action-specific effects proposed by Molto et al. (2020). Therefore, we concluded that the effect of barriers on distance perception is robust and observable in real and virtual environments.

\section{Action-Specific Effects in a Virtual Environment}

The experiments of the present study were conducted in a virtual environment. Using virtual reality facilitates the study of behavioral and neural responses in difficult or impossible situations to implement in the real environment. Therefore, many studies investigating action-specific effects have used virtual reality techniques (e.g., Stefanucci et al., 2005; Laitin et al., 2019). We successfully replicated the findings of Morgado et al. (2013) in a virtual environment, and we believe that virtual reality is an essential tool for investigating action-specific effects. However, there are some differences between real and virtual environments that can affect visual perception. One critical difference is that people underestimate egocentric distances in virtual environments compared to real environments (see Renner et al., 2013, for a review). This distance compression effect might influence people's behavioral responses. In Morgado et al. (2013), the interaction between the barrier's width and the actual distance to the target on distance estimation was marginally significant. The effect of the barrier width was not observed in the position nearest to the target of $34 \mathrm{~cm}$, although it was observed in the three longer target position conditions of $37 \mathrm{~cm}, 40 \mathrm{~cm}$, and $43 \mathrm{~cm}$. If the distance to a target affects action-specific effects, then distance compression might reduce the effect of a barrier in the virtual environment compared to the real environment because the target could be perceived as identical in both environments. The effect size of the barrier width was relatively large in the visible condition of Experiment 1, although the magnitude of the overestimated distance was much smaller than in Morgado et al. (2013). The mean overestimated distance difference between a narrow and wide barrier was $5.77 \mathrm{~mm}$ in the visible condition of this study, whereas it was approximately $12 \mathrm{~mm}$ in Morgado et al. (2013). The smaller difference in the overestimated distances between a narrow and a wide barrier could be explained by distance compression caused by the virtual environment. In sum, virtual reality is a powerful experimental tool. However, it should be noted that there are specific perceptual differences between the real and a virtual environment. The failure to consider these differences might result in decreased sensitivity or replication failures using virtual reality environments.

\section{Limitations and Conclusion}

There are specific limitations to this study. Firstly, we did not consider individual differences in stereoscopic vision on actionspecific effects. A previous study suggests that nearly $30 \%$ of the population does not have normal stereoscopic vision (Hess et al., 2015). Stereoscopic vision is an essential ability for estimating the egocentric distance to an object. Therefore, the effect of individual differences in stereoscopic vision on distance estimation and action-specific effects should be examined in future studies. Secondly, we instructed participants to imagine reaching directly at the target by penetrating a visible but untouchable barrier. In Experiment 2, participants could also choose to reach around the barrier at the target, although this was not the most economical action. In the present study, we focused on the effect of action imagination on distance perception when people imagined an action with the lowest costs under a given situation. However, this manipulation made it difficult to directly compare Experiments 1 and 2 because two variables differed between the two experiments; the presence of tactile feedback about the barrier and action imagination instructions. Therefore, more detailed experiments should be undertaken in the future to examine the effect of each factor affecting action-specific effects. Finally, in the present study, the participants estimated the distance to the target by visually matching the distances at different angles. This experimental paradigm is not commonly used in distance estimation research, although several studies have used this (Osiurak et al., 2012; Morgado et al., 2013). Then, more studies are needed to establish the validity of this method.

We investigated tactile feedback on obstacles and related action imagery on action-specific effects. We also attempted to replicate Morgado et al. (2013), which was accomplished in this study. However, studies on action-specific effects have issues other than replicability. For example, several studies have indicated insufficient theoretical explanations of action-specific effects. It is also possible that action-specific effects largely reflect experimenter demands (Durgin et al., 2009; Firestone and Scholl, 2016). The lack of theoretical background is a serious shortcoming that should be overcome in future studies on action-specific effects. Actionspecific effects are induced by some action constraints or extensions such as weight on the body, action effort, or tooluse, and these constraints or extensions are also related to the expansion or contraction of the peripersonal space (weight on the body: Lourenco and Longo, 2009, tool-use: Iriki et al., 1996; Holmes et al., 2004; Longo and Lourenco, 2006). The peripersonal space is a multisensory spatial representation surrounding a person's body or body parts that support the person's actions through their body. Examining the relationship between action-specific effects and the peripersonal space could lead to further understanding of action-specific effects. It is suggested that future studies investigate this issue. 


\section{DATA AVAILABILITY STATEMENT}

The raw data supporting the conclusions of this article will be made available by the authors, without undue reservation.

\section{ETHICS STATEMENT}

The studies involving human participants were reviewed and approved by Department of Psychology in the University of Tokyo. The patients/participants provided their written informed consent to participate in this study.

\section{REFERENCES}

Afonso, A., Blum, A., Katz, B. F. G., Tarroux, P., Borst, G., and Denis, M. (2010). Structural Properties of Spatial Representations in Blind People: Scanning Images Constructed from Haptic Exploration or from Locomotion in a 3-D Audio Virtual Environment. Mem. Cogn. 38, 591-604. doi:10.3758/MC.38.5.591

Bhalla, M., and Proffitt, D. R. (1999). Visual-motor Recalibration in Geographical Slant Perception. J. Exp. Psychol. Hum. Perception Perform. 25, 1076-1096. doi:10.1037/0096-1523.25.4.1076

Cohen, R. G., Biddle, J. C., and Rosenbaum, D. A. (2010). Manual Obstacle Avoidance Takes into Account Visual Uncertainty, Motor Noise, and Biomechanical Costs. Exp. Brain Res. 201, 587-592. doi:10.1007/s00221-0092042-8

Costello, M. C., Bloesch, E. K., Davoli, C. C., Panting, N. D., Abrams, R. A., and Brockmole, J. R. (2015). Spatial Representations in Older Adults Are Not Modified by Action: Evidence from Tool Use. Psychol. Aging 30, 656-668. doi:10.1037/pag0000029

Creem-Regehr, S. H., and Kunz, B. R. (2010). Perception and Action. Wires Cogn. Sci. 1, 800-810. doi:10.1002/wcs.82

Durgin, F. H., Baird, J. A., Greenburg, M., Russell, R., Shaughnessy, K., and Waymouth, S. (2009). Who Is Being Deceived? the Experimental Demands of Wearing a Backpack. Psychon. Bull. Rev. 16, 964-969. doi:10.3758/PBR.16.5.964

Firestone, C., and Scholl, B. J. (2016). Cognition Does Not Affect Perception: Evaluating the Evidence for "Top-Down" Effects. Behav. Brain Sci. 39. doi:10.1017/S0140525X15000965

Hess, R. F., To, L., Zhou, J., Wang, G., and Cooperstock, J. R. (2015). Stereo Vision: The Haves and Have-Nots. i-Perception 6, 204166951559302. doi:10.1177/ 2041669515593028

Holmes, N. P., Calvert, G. A., and Spence, C. (2004). Extending or Projecting Peripersonal Space with Tools? Multisensory Interactions Highlight Only the Distal and Proximal Ends of Tools. Neurosci. Lett. 372, 62-67. doi:10.1016/ j.neulet.2004.09.024

Hutchison, J. J., and Loomis, J. M. (2006). Does Energy Expenditure Affect the Perception of Egocentric Distance? A Failure to Replicate Experiment 1 of Proffitt, Stefanucci, Banton, and Epstein (2003). Span. J. Psychol. 9, 332-339. doi:10.1017/S1138741600006235

Kirsch, W., Herbort, O., Butz, M. V., and Kunde, W. (2012). Influence of Motor Planning on Distance Perception within the Peripersonal Space. PLoS One 7, e34880. doi:10.1371/journal.pone.0034880

Lahav, O., and Mioduser, D. (2008). Haptic-feedback Support for Cognitive Mapping of Unknown Spaces by People Who Are Blind. Int. J. HumanComputer Stud. 66, 23-35. doi:10.1016/j.ijhcs.2007.08.001

Laitin, E. L., Tymoski, M. J., Tenhundfeld, N. L., and Witt, J. K. (2019). The Uphill Battle for Action-specific Perception. Atten Percept Psychophys 81, 778-793. doi:10.3758/s13414-018-01652-w

Lessard, D. A., Linkenauger, S. A., and Proffitt, D. R. (2009). Look before You Leap: Jumping Ability Affects Distance Perception. Perception 38, 1863-1866. doi:10.1068/p6509

Linkenauger, S. A., Bülthoff, H. H., and Mohler, B. J. (2015). Virtual Arm's Reach Influences Perceived Distances but Only after Experience Reaching. Neuropsychologia 70, 393-401. doi:10.1016/j.neuropsychologia.2014.10.034

\section{AUTHOR CONTRIBUTIONS}

All authors listed have made a substantial, direct, and intellectual contribution to the work and approved it for publication.

\section{FUNDING}

This work was supported by the Japan Society for the Promotion of Science (Grant numbers: 19H01490) to KY. The funders had no role in study design, data collection, and analysis, the decision to publish, or manuscript preparation.

Longo, M. R., and Lourenco, S. F. (2006). On the Nature of Near Space: Effects of Tool Use and the Transition to Far Space. Neuropsychologia 44, 977-981. doi:10.1016/j.neuropsychologia.2005.09.003

Lourenco, S. F., and Longo, M. R. (2009). The Plasticity of Near Space: Evidence for Contraction. Cognition 112, 451-456. doi:10.1016/j.cognition.2009.05.011

lriki, A., Tanaka, M., and Iwamura, Y. (1996). Coding of Modified Body Schema during Tool Use by Macaque Postcentral Neurones. Neuroreport 7, 2325-2330. doi:10.1097/00001756-199610020-00010

Mine, D., Ogawa, N., Narumi, T., and Yokosawa, K. (2020). The Relationship between the Body and the Environment in the Virtual World: The Interpupillary Distance Affects the Body Size Perception. PLoS One 15, e0232290-19. doi:10.1371/journal.pone.0232290

Molto, L., Nalborczyk, L., Palluel-Germain, R., and Morgado, N. (2020). Action Effects on Visual Perception of Distances: A Multilevel Bayesian Meta-Analysis. Psychol. Sci. 31, 488-504. doi:10.1177/0956797619900336

Morash, V., Connell Pensky, A. E., Alfaro, A. U., and McKerracher, A. (2012). A Review of Haptic Spatial Abilities in the Blind. Spat. Cogn. Comput. 12, 83-95. doi:10.1080/13875868.2011.599901

Morgado, N., Gentaz, É., Guinet, É., Osiurak, F., and Palluel-Germain, R. (2013). Within Reach but Not So Reachable: Obstacles Matter in Visual Perception of Distances. Psychon. Bull. Rev. 20, 462-467. doi:10.3758/s13423-012-0358-Z

Ogawa, N., Narumi, T., and Hirose, M. (2017). Distortion in Perceived Size and Body-Based Scaling in Virtual Environments. ACM Int. Conf. Proceeding Ser. doi:10.1145/3041164.3041204

Osiurak, F., Morgado, N., and Palluel-Germain, R. (2012). Tool Use and Perceived Distance: When Unreachable Becomes Spontaneously Reachable. Exp. Brain Res. 218, 331-339. doi:10.1007/s00221-012-3036-5

Philbeck, J. W., and Witt, J. K. (2015). Action-Specific Influences on Perception and Postperceptual Processes: Present Controversies and Future Directions. Psychol. Bull. 141, 1120-1144. doi:10.1037/a0039738

Proffitt, D. R., and Linkenauger, S. A. (2013). Perception Viewed as a Phenotypic Expression. Action. Sci., 171-197. doi:10.7551/mitpress/ 9780262018555.003.0007

Proffitt, D. R., Stefanucci, J., Banton, T., and Epstein, W. (2006). Reply to Hutchison and Loomis. Span. J. Psychol. 9, 340-342. doi:10.1017/ S1138741600006247

Proffitt, D. R., Stefanucci, J., Banton, T., and Epstein, W. (2003). The Role of Effort in Perceiving Distance. Psychol. Sci. 14, 106-112. doi:10.1111/1467-9280.t01-101427

Quarona, D., Raffuzzi, M., Costantini, M., and Sinigaglia, C. (2020). Preventing Action Slows Down Performance in Perceptual Judgment. Exp. Brain Res. 238, 2857-2864. doi:10.1007/s00221-020-05948-y

Renner, R. S., Velichkovsky, B. M., and Helmert, J. R. (2013). The Perception of Egocentric Distances in Virtual Environments - A Review. ACM Comput. Surv. 46, 1-40. doi:10.1145/2543581.2543590

Stefanucci, J. K., Proffitt, D. R., Banton, T., and Epstein, W. (2005). Distances Appear Different on hills. Perception \& Psychophysics 67, 1052-1060. doi:10.3758/BF03193631

Tosi, G., Parmar, J., Dhillon, I., Maravita, A., and Iaria, G. (2020). Body Illusion and Affordances: the Influence of Body Representation on a Walking Imagery Task in Virtual Reality. Exp. Brain Res. 238, 2125-2136. doi:10.1007/s00221-02005874-Z 
van der Hoort, B., Guterstam, A., and Ehrsson, H. H. (2011). Being Barbie: The Size of One's Own Body Determines the Perceived Size of the World. PLoS One 6, e20195. doi:10.1371/journal.pone.0020195

Witt, J. K., and Proffitt, D. R. (2008). Action-Specific Influences on Distance Perception: A Role for Motor Simulation. J. Exp. Psychol. Hum. Perception Perform. 34, 1479-1492. doi:10.1037/a0010781

Witt, J. K., Proffitt, D. R., and Epstein, W. (2004). Perceiving Distance: A Role of Effort and Intent. Perception 33, 577-590. doi:10.1068/p5090

Witt, J. K., Proffitt, D. R., and Epstein, W. (2005). Tool Use Affects Perceived Distance, but Only when You Intend to Use it. J. Exp. Psychol. Hum. Perception Perform. 31, 880-888. doi:10.1037/0096-1523.31.5.880

Witt, J. K., Schuck, D. M., and Taylor, J. E. T. (2011). Action-specific Effects Underwater. Perception 40, 530-537. doi:10.1068/p6910

Witt, J. K. (2011). Tool Use Influences Perceived Shape and Perceived Parallelism, Which Serve as Indirect Measures of Perceived Distance. J. Exp. Psychol. Hum. Perception Perform. 37, 1148-1156. doi:10.1037/ a0021933

Woods, A. J., Philbeck, J. W., and Danoff, J. V. (2009). The Various Perceptions of Distance: An Alternative View of How Effort Affects
Distance Judgments. J. Exp. Psychol. Hum. Perception Perform. 35, 1104-1117. doi:10.1037/a0013622

Conflict of Interest: The authors declare that the research was conducted in the absence of any commercial or financial relationships that could be construed as a potential conflict of interest.

Publisher's Note: All claims expressed in this article are solely those of the authors and do not necessarily represent those of their affiliated organizations, or those of the publisher, the editors and the reviewers. Any product that may be evaluated in this article, or claim that may be made by its manufacturer, is not guaranteed or endorsed by the publisher.

Copyright (C) 2021 Mine, Kimoto and Yokosawa. This is an open-access article distributed under the terms of the Creative Commons Attribution License (CC BY). The use, distribution or reproduction in other forums is permitted, provided the original author(s) and the copyright owner(s) are credited and that the original publication in this journal is cited, in accordance with accepted academic practice. No use, distribution or reproduction is permitted which does not comply with these terms. 